\title{
Continuous Criterion for Parallel MRI Reconstruction Using B-spline Approximation (PROBER)
}

\author{
Jan Petr, Jan Kybic \\ Center for Machine Perception, Dpt. of Cybernetics, Faculty of Electrical Engineering, \\ Czech Technical University in Prague, Karlovo nám. 13, 12135 Praha 2, Czech Republic
}

\begin{abstract}
Parallel MRI is a way to use multiple receiver coils with distinct spatial sensitivities to increase the speed of the MRI acquisition. The acquisition is speeded up by undersampling in the phase-encoding direction and the resulting data loss and aliasing is compensated for by the use of the additional information obtained from several receiver coils.

The task is to reconstruct an unaliased image from a series of aliased images. We have proposed an algorithm called PROBER that takes advantage of the smoothness of the reconstruction transformation in space. B-spline functions are used to approximate the reconstruction transformation. Their coefficients are estimated at once minimizing the total expected reconstruction error. This makes the reconstruction less sensitive to noise in the reference images and areas without signal in the image. We show that this approach outperforms the SENSE and GRAPPA reconstruction methods for certain coil configurations.

In this article, we propose another improvement, consisting of a continuous representation of the B-splines to evaluate the error instead of the discretely sampled version. This solves the undersampling issues in the discrete B-spline representation and offers higher reconstruction quality which has been confirmed by experiments. The method is compared with the discrete version of PROBER and with commercially used algorithms GRAPPA and SENSE in terms of artifact suppression and reconstruction SNR.
\end{abstract}

Keywords: Image quality, Restoration, Magnetic resonance, B-splines

\section{INTRODUCTION}

Parallel MRI is a technique that exploits the intrinsic spatial encoding of multiple receiver coils (array coils) with distinct spatial sensitivities to speed up MRI data acquisition. ${ }^{1-3}$ This decreases the total time of patient examination, helps to avoid motion artifacts and increases frame rate when visualizing dynamic processes. The increase in data acquisition speed is achieved by sampling the $k$-space more sparsely in the phase encoding direction. The image information is not retrieved completely which causes aliasing in the images. The missing $k$-space information is synthesized using the sensitivity information from multiple receiver coils.

Several reconstruction algorithms have been described working either in the $k$-space (frequency domain) or in the spatial domain. The first $k$-space method described is the Simultaneous Acquisition of Spatial Harmonics (SMASH). ${ }^{1}$ In SMASH, the missing phase-encoding steps are reconstructed directly in the $k$-space as a linear combination of the neighboring phase-encoding lines. The auto-calibration process was later introduced ${ }^{2,4}$ using fully sampled portions of the $k$-space for calibration without the need for a separate acquisition to extract the sensitivity maps. The second group of reconstruction methods works in the spatial domain. Sensitivity encoding (SENSE) ${ }^{3}$ uses extracted array-coil sensitivities to build an encoding matrix. This matrix transforms the unaliased image without sensitivity modulation to aliased array-coil images. The reconstruction matrix is obtained by inverting the encoding matrix. In the case of Cartesian sampling, only few pixels contribute to each aliased pixel. Thus, the matrix inversion can be done separately for each pixel.

We have introduced a novel imaged-based reconstruction method Parallel MRI Reconstruction Using B-spline Approximation (PROBER) $)^{5,6}$ which takes advantage of the linearity and of the smoothness of the reconstruction transformation. The reconstruction transformation is estimated directly using raw images by minimizing an error

email: petrj5@cmp.felk.cvut.cz; kybic@cmp.felk.cvut.cz 
criterion. This criterion penalizes the presence of aliasing artifacts and noise in the reconstructed image. The reconstruction transformation is represented in a B-spline basis. ${ }^{7}$ This reduces the number of reconstruction coefficients, regularizes the reconstruction and makes it more stable. In this article, the PROBER method is improved by using a continuous $L_{2}$ norm to evaluate the error instead the discrete $l_{2}$ norm. This improves the estimation quality and makes the reconstruction more precise. The new method is compared with GRAPPA ${ }^{2}$ (a variation of SMASH) and $\mathrm{SENSE}^{3}$ in terms of aliasing removal and reconstruction noise level.

\section{PARALLEL MRI}

In parallel MRI, the signal is acquired using several receiver coils (array coils). The array-coil image $S_{l}$ obtained from the $l$-th receiver coil is a pointwise product of the original image $S$ and the coil sensitivities $C_{l}$

$$
S_{l}(x, y)=C_{l}(x, y) S(x, y) .
$$

The input images are downsampled in the $k$-space to increase the acquisition speed. This causes aliasing in the phase-encoding direction (here, the $y$ direction). The relation between the aliased array-coil images $S_{l}^{A}$ and the fully-sampled image $S$ is ${ }^{8}$

$$
S_{l}^{A}(x, y)=\sum_{m=0}^{M-1} S\left(x, y \bmod \frac{Y}{M}+m \frac{Y}{M}\right) C_{l}\left(x, y \bmod \frac{Y}{M}+m \frac{Y}{M}\right),
$$

where $M$ is the acceleration factor (i.e. every $M$-th $k$-space line is retrieved) and $Y$ is the original image size in the phase-encoding direction.

The task of parallel MRI is to reconstruct an unaliased image $\hat{S}$ approximating the original image $S$ from a set of input images $S_{l}^{A}$ (aliased array-coil images). The reconstruction transformation $\alpha_{l}$ is linear because of the linearity of (1) and (2)

$$
\hat{S}(x, y)=\sum_{l=1}^{L} \alpha_{l}(x, y) S_{l}^{A}(x, y),
$$

where the weights $\alpha_{l}$ are estimated using a set of reference images $S, S_{l}$. Reference images are aliasing free, can be low-resolution, and can be acquired for a different object than the object to be reconstructed.

\section{RECONSTRUCTION USING B-SPLINE APPROXIMATION}

In this section we summarize the discrete PROBER method. ${ }^{5,6}$ The reconstruction weights $\alpha_{l}(3)$ are obtained by minimizing the expected reconstruction error. The error is evaluated as using a discrete $l_{2}$ norm on reference images $S, S_{l}$

$$
e=\sum_{m, m^{\prime}=0}^{M-1}\left\|\sum_{l=1}^{L} \alpha_{l, m} S_{l, m^{\prime}}-\delta_{m, m^{\prime}} S_{m}\right\|_{l_{2}}^{2}+\sum_{l=1}^{L} \sigma_{l}^{2}\left\|\alpha_{l}\right\|_{l_{2}}^{2},
$$

where $\delta_{m, m^{\prime}}$ is Kronecker delta, $\sigma_{l}^{2}$ is the variance of Gaussian sensor noise for the $l$-th coil and the $l_{2}$ norm $\|\ldots\|_{l_{2}}^{2}$ is over all pixels $(x, y)$. We have used a shortened notation: $S_{l}$ resp. $S_{l, m}$ is used instead of $S_{l}(x, y)$ resp. $S_{l}\left(x, y \bmod \frac{Y}{M}+m \frac{Y}{M}\right)$ and similarly for $\alpha_{l, m}$. The first term in (4) describes the aliasing and the reconstruction error and the second term corresponds to the noise variance in the reconstructed image.

B-spline approximation of the reconstruction weights $\alpha_{l}$ is introduced in order to decrease the number of unknowns and, thus, regularize and speed up the estimation process. Tensor products of evenly spaced and scaled B-splines ${ }^{7} \varphi_{i}(y), \varphi_{j}(x)$ are used as basis functions to approximate the weights $\alpha_{l}$

$$
\alpha_{l}(x, y)=\sum_{i=1}^{I} \sum_{j=1}^{J} g_{i j l} \varphi_{i}(y) \varphi_{j}(x),
$$


where $I$ resp. $J$ is the number of B-spline functions in the $y$ resp. $x$ direction. The B-spline coefficients $g_{i j l}$ are sought by minimizing the error criterion (4).

Low-resolution reference images are often used for the estimation. The B-spline functions $\varphi_{i}(y)$ and $\varphi_{j}(x)$ are then sampled in low resolution to evaluate the reconstruction error (4) and resampled in full resolution to obtain the weights $\alpha_{l}$ (5) for the reconstruction (3). Thus the estimated reconstruction transformation is upsampled using B-spline interpolation. However, low resolution reference images are obtained as a Fourier transform of the $k$-space center - which is equivalent to interpolation using harmonic functions. This discrepancy reduces the accuracy of estimation. We propose to use the $L_{2}$ norm to evaluate the error to remedy the situation.

\section{CONTINUOUS CRITERION}

\subsection{Continuous representation of reference images}

In order to evaluate the error criterion (4) as continuous, it is necessary to use a continuous representation of reference images. We convert the images $S_{l}$ into a continuous B-spline representation. B-splines are real-valued and spatially localized which is advantageous for the speed of the estimation process as described later. The additional time to convert the images to the B-spline representation is negligible when compared with the total estimation time.

Reference images are represented as continuous in the B-spline basis $\psi_{p}(y), \psi_{q}(x)$ with coefficients $h_{p q l}$

$$
{ }^{c} S_{l}(x, y)=\sum_{p, q=1}^{P, Q} h_{p q l} \psi_{p}(y) \psi_{q}(x),
$$

where $P$ resp. $Q$ is the number of B-splines used to represent the image in the $y$ resp. $x$ direction. Similarly to $\varphi_{i}(y)$ and $\varphi_{j}(x),{ }^{6} \mathrm{~B}$-splines are equally spaced over the image

$$
\begin{aligned}
& \psi_{p}(y)=b_{v_{p}}^{3}(y-p)=\beta^{3}\left(y / v_{p}-p\right), \\
& \psi_{q}(x)=b_{v_{q}}^{3}(x-q)=\beta^{3}\left(x / v_{q}-q\right),
\end{aligned}
$$

where $\beta^{3}$ is a cubic B-spline function ${ }^{9}$ and $b_{v}^{3}$ is a scaled cubic B-spline. The scaling $v_{p}, v_{q}$ is defined as $v_{p}=Y / P$, $v_{q}=X / Q$ where $Y \times X$ is the reference image size. The B-spline spacing of 1 pixel $(Y=P)$ is normally used to ensure accurate representation. However, the number of splines can be decreased to decrease the time complexity of the estimation process - see Section 5.2.

The B-spline coefficients can be obtained by recursive filtering in the image domain. ${ }^{9,10}$ Since the input images are given in the $k$-space $\left(S_{l}(x, y)=\mathcal{D} \mathcal{F} \mathcal{T}\left\{s_{l}\left(k_{x}, k_{y}\right)\right\}\right)$ it is more precise and efficient to apply the inverse $\mathrm{B}$-spline filter directly in the $k$-space. The Fourier transformation of the inverse filter $\left(\mathrm{B}_{v}^{3}\right)^{-1}(f)$ is ${ }^{9}$

$$
\left(\mathrm{B}_{v}^{3}\right)^{-1}(f)=v^{3}\left(\frac{\sin (\pi f)}{\sin (\pi v f)}\right)\left(b_{1}^{3}(0)+\sum_{k=1}^{2} 2 b_{1}^{3}(k) \cos (2 \pi f k)\right)^{-1} .
$$

Using separability, the B-spline coefficients $h_{p q l}$ are obtained as ${ }^{10,11}$

$$
h_{p q l}=\mathcal{D} \mathcal{F} \mathcal{T}_{x}\left\{\mathcal{D F} \mathcal{T}_{y}\left\{s\left(k_{x}, k_{y}\right) \cdot\left(\mathrm{B}_{\mathrm{v}_{\mathrm{p}}}^{3}\right)^{-1}\left(k_{y}\right)\right\} \cdot\left(\mathrm{B}_{\mathrm{v}_{\mathrm{q}}}^{3}\right)^{-1}\left(k_{x}\right)\right\} .
$$




\subsection{Continuous error}

The continuous error criterion is derived from the discrete criterion (4) by replacing the $l_{2}$ norm with the $L_{2}$ norm and by replacing the discrete reference images $S_{l}$ with their continuous version ${ }^{c} S_{l}(6)$

$$
\begin{aligned}
e & =\sum_{m, m^{\prime}=0}^{M-1}\left\|\sum_{l=1}^{L} \alpha_{l, m}{ }^{c} S_{l, m^{\prime}}-\delta_{m, m^{\prime}}{ }^{c} S_{m}\right\|_{L_{2}}^{2}+\sum_{l=1}^{L} \sigma_{l}^{2}\left\|\alpha_{l}\right\|_{L_{2}}^{2} \\
& =\sum_{m, m^{\prime}=0}^{M-1} \int_{x, y}\left|\sum_{l=1}^{L} \alpha_{l, m} \sum_{p, q=1}^{P, Q} h_{p q l} \psi_{p, m^{\prime}} \psi_{q}-\delta_{m, m^{\prime}} \sum_{p, q=1}^{P, Q} h_{p q} \psi_{p, m} \psi_{q}\right|^{2}+\sum_{l=1}^{L} \sigma_{l}^{2}\left|\alpha_{l}\right|^{2} \mathrm{~d} x \mathrm{~d} y \\
& =\sum_{m, m^{\prime}} \int_{x, y}\left|\sum_{i, j, l} g_{i j l} \varphi_{i, m} \varphi_{j} \sum_{p, q} h_{p q l} \psi_{p, m^{\prime}} \psi_{q}-\delta_{m, m^{\prime}} \sum_{p, q} h_{p q} \psi_{p, m} \psi_{q}\right|^{2}+\sum_{l} \sigma_{l}^{2}\left|\sum_{i, j} g_{i j l} \varphi_{i} \varphi_{j}\right|^{2} \mathrm{~d} x \mathrm{~d} y,
\end{aligned}
$$

where $\psi_{p}, \psi_{q}$ are the B-spline basis used for the reference image representation (7).

Minimization of the reconstruction error (9) leads to a set of $I \cdot J \cdot L$ linear equations $\mathbf{B}=\mathbf{A g}$. Matrices $\mathbf{A}$ and $\mathbf{B}$ are given by

$$
\begin{aligned}
\mathbf{A}_{\left[i j l ; i^{\prime} j^{\prime} l^{\prime}\right]} & =\sum_{p p^{\prime}} \Psi^{y}\left(p, p^{\prime}, i, i^{\prime}\right) \sum_{q q^{\prime}} h_{p^{\prime} q^{\prime} l^{\prime}} h_{p q l}^{*} \Psi^{x}\left(q, q^{\prime}, j, j^{\prime}\right)+\sigma_{l}^{2} \int_{x, y} \varphi_{i} \varphi_{i^{\prime}} \varphi_{j} \varphi_{j^{\prime}} \mathrm{d} x \mathrm{~d} y \\
\mathbf{B}_{[i j l]} & =\sum_{p p^{\prime}} \Phi^{y}\left(p, p^{\prime}, i\right) \sum_{q q^{\prime}} h_{p^{\prime} q^{\prime}} h_{p q l}^{*} \Phi^{x}\left(q, q^{\prime}, j\right),
\end{aligned}
$$

where $\Psi^{y}, \Psi^{x}, \Phi^{y}$ and $\Phi^{x}$ are B-spline kernels corresponding to the scalar product of the B-spline basis functions. ${ }^{12}$ The matrix $\mathbf{A}$ is Hermitian and positive-definite. ${ }^{6}$ The system $\mathbf{B}=\mathbf{A g}$ can be solved using Cholesky decomposition. ${ }^{13}$ See Appendix A for details.

The PROBER estimation using the continuous criterion (9) avoids the problems caused by undersampling of the B-splines during the estimation with low-resolution images. It allows more precise evaluation of the reconstruction error which improves the overall quality of the reconstruction.

\section{RESULTS}

We have acquired 30 sets of phantom and in-vivo images on a clinical 1.5T MR system with 32 receiver channels (Magnetom Avanto, Siemens Medical Solutions, Erlangen, Germany) using a gradient echo pulse sequence. ${ }^{14}$ Variable-density scans were acquired with a fully sampled $k$-space center (24 lines). The $k$-space center serves as the low-resolution reference image. The image size is $256 \times 256$ pixels for datasets $1,3,5,7,9-12,14-20,22-30$ and $512 \times 512$ pixels for the rest of the datasets. Datasets $12-16$ and 19 were acquired using 5 coils; 6 coils were used for sets 17 and $18 ; 8$ coils for sets $1-11$ and 20-24; 14 coils for sets 25 and $26 ; 17$ coils for sets $27-28$ and 24 coils for sets 29-30.

Plexi-glass phantoms filled with a 1:100 Gd-DTPA:water solution were used. The first eleven datasets were acquired with an 8-channel head coil and a phantom that was designed to measure image distortion. In datasets 12-18, a vessel phantom is visualized using a 6-channel spine array coil. To asses the performance of the methods on real images, 12 sets of in-vivo head and body images of a 25 year old healthy volunteer were obtained. Two sets of transversal (sets 20 and 21) and three sets of sagittal head images at the mid-line (sets 24-26) were acquired. The sets 19, 23, 27-30 contain coronal and the set 22 transversal images of the torso.

Ground truth images and sensitivity maps were extracted from 11 sets of real images (corresponding to sets $8,13,21,18,19,23,24,10,11,25$ and 27$)$ to be used to generate simulated images. The ground truth image was obtained as a sum-of-squares of the array-coil images. The intensity bias caused by the sum-of-squares combination was corrected using a non-parametric intensity correction method ${ }^{15}$ and normalized to mean 0.5 . Sensitivity maps were obtained by dividing the array-coil images by the ground truth image. The maps were filtered to remove noise. ${ }^{3}$ The ground truth images and sensitivity maps were used to generate simulated images 
with size $256 \times 256$ pixels. We added white additive Gaussian noise with noise variance from $10^{-4}$ to $10^{-3}$ to the both real and imaginary components. The advantage of the simulated images is that we can control noise variance in the images and the ground truth image is known. Thus, we can evaluate the reconstruction quality more precisely.

\subsection{Reconstruction error}

We observe two types of errors in the reconstructed images - aliasing artifacts and noise. Aliasing artifacts are the deterministic part of the error. To quantify these artifacts we take the mean $\bar{S}$ of 20 reconstructions $\hat{S}_{k}$. The mean $\bar{S}$ is obtained as $\bar{S}=\sum_{k=1}^{20} \hat{S}^{k} / 20$. We calculate the artifact power (AP) ${ }^{16}$ as

$$
\mathrm{AP}=\frac{\|\bar{S}-S\|_{x, y}^{2}}{\|S\|_{x, y}^{2}}
$$

where $S$ is the true image. Noise in the reconstruction is quantified using SNR

$$
\mathrm{SNR}=20 \log _{10} \sqrt{\sum_{k=1}^{20} \frac{\|S\|_{x, y}^{2}}{20\left\|\hat{S}^{k}-\bar{S}\right\|_{x, y}^{2}}} .
$$

It is not possible to obtain the mean image $\bar{S}$ for the real measurements due to motion artifacts. Thus, we compute only the SNR in this case

$$
\mathrm{SNR}_{\text {real }}=20 \log _{10} \sqrt{\frac{\|S\|_{x, y}^{2}}{\|\hat{S}-S\|_{x, y}^{2}}} .
$$

The disadvantage is that $\mathrm{SNR}_{\text {real }}$ is affected by both aliasing artifacts and noise. Moreover, the ground truth image $S$ is obtained using only one set of fully sampled images. Therefore, it contains noise which makes it less appropriate for quantitative error evaluation.

\subsection{Number of Splines}

The number of B-splines used to represent the reference images is an important factor for quality of the reconstruction. We have evaluated the reconstruction quality (using equation (13)) on simulated images with varying number of B-splines (using the settings $M=2, \sigma_{l}^{2}=10^{-4}, I=J=8, P=Q=6, \ldots, 24$ ), Figure 1 . The reconstruction SNR are normalized by setting the maximal SNR value to 1 for each set. The mean SNR over all the simulated sets is shown in Figure 1a.

The quality for less than $10 \times 10$ B-splines is low due to strong aliasing artifacts - see Figure 1c. The reconstructions are free of artifacts for more than $16 \times 16$ splines. The SNR for $16 \times 16$ B-splines is only $4 \%$ lower than maximal value. In the following experiments we have chosen $20 \times 20$ B-splines for optimal quality with reconstruction times comparable to SENSE and GRAPPA. However, $16 \times 16$ B-splines can be used without significant degradation of quality with the benefit of increased reconstruction speed.

\subsection{Experimental comparison}

The results of the continuous and discrete PROBER method were compared with $\mathrm{SENSE}^{3}$ and GRAPPA ${ }^{2}$ on simulated and real images for acceleration factors 2 and 4. Signal-to-noise ratio (12) and artifact power (11) were used for the quantitative evaluation.

The methods were tested first on the simulated data sets for acceleration factor 2 and noise variance $10^{-4}$ and $10^{-3}$ - see Figure 2. For input images with small noise variance $\left(\sigma_{l}^{2}=10^{-4}\right)$, PROBER has the lowest AP of all methods on all images. The PROBER SNR is higher than the GRAPPA SNR, and higher or not more than 1\% lower than the SENSE SNR on all images. See Figure 3 for reconstruction of simulated dataset 10. The continuous PROBER version reaches better results than the discrete version on majority of the images. For simulated images with high noise variance $\left(\sigma_{l}^{2}=10^{-3}\right)$, PROBER reaches better artifact suppression than 


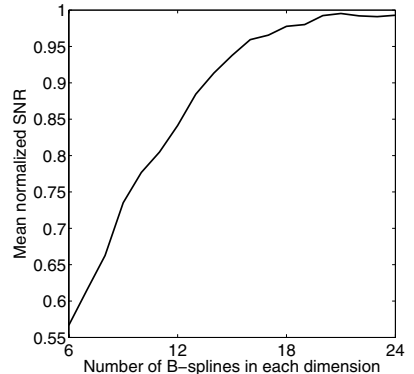

a) Mean normalized SNR

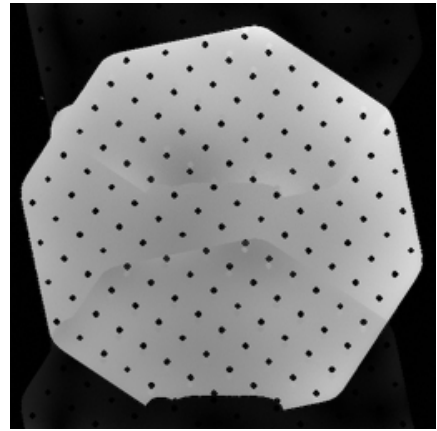

c) Set 1, $6 \times 6$ B-splines

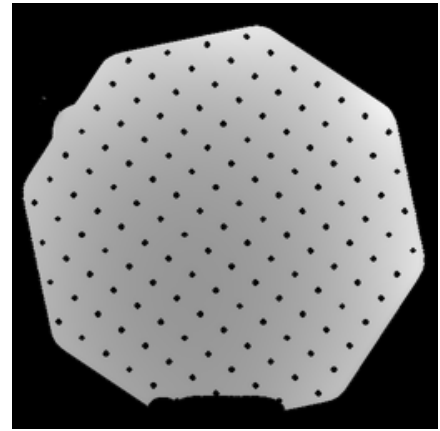

b) Set 1 , original image

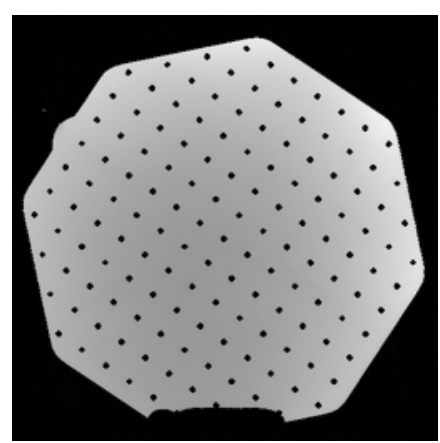

d) Set $1,16 \times 16$ B-splines

Figure 1. PROBER was tested for various number of B-splines $(P, Q)$ used to represent the reference images. The normalized SNR averaged over all simulated sets is shown in $(a)$. The reconstruction for set 1: (b) original, $(c) 6 \times 6$ B-splines - aliasing artifacts, (d) $16 \times 16$ B-splines.

SENSE and GRAPPA with similar SNR on most of the images. SENSE reaches the best results regarding both AP and SNR on sets 1, 3 and 8. PROBER and SENSE reach the same quality on set 9 while on set 11, PROBER and GRAPPA achieve similar results. The continuous PROBER version has lower AP than the discrete version a on half of the data, whereas the SNR of the continuous PROBER method is slightly better than the SNR of the discrete version on all images. We conclude that the PROBER method offers the best artifact suppression out of the three tested methods on most of the simulated images. The reconstruction noise level is similar to the other methods (as is clear from the reconstruction SNR).

The results on simulated datasets for the acceleration factor 4 and noise variance $\sigma_{l}^{2}=5 \cdot 10^{-4}$ are in Figure 4. The reconstruction for acceleration factor 4 is often ill-conditioned due to unfavorable coil configuration. Therefore, the quantitative results need to be interpreted with care. PROBER has the lowest SNR and AP for sets 8, 9 and 11 (see Figure 5), whereas GRAPPA for sets 4 and 5. PROBER and GRAPPA show significantly better results than SENSE for sets 2, 6, 7 and 10. No method can be identified as the best for sets 1 and 3 . The results on simulated images with acceleration factor 4 shows that the PROBER reconstruction can reach reasonable results on images where the GRAPPA and SENSE methods produce only moderate quality results.

The results reached on simulated images are confirmed on real data (Figure 6). Only the SNR is used to quantify the reconstruction quality - see equation (13). Since noise free ground truth images are not available, the results on real images should be regarded as indication only. The PROBER SNR is higher than the GRAPPA and SENSE SNR on all phantom images except datasets 8 and 17. PROBER reaches better or comparable SNR than GRAPPA on all in-vivo images and significantly better SNR than SENSE on all in-vivo images except datasets 20 and 21. The continuous PROBER shows better SNR than the discrete version on the phantom images, on in-vivo images the methods reach comparable results. 


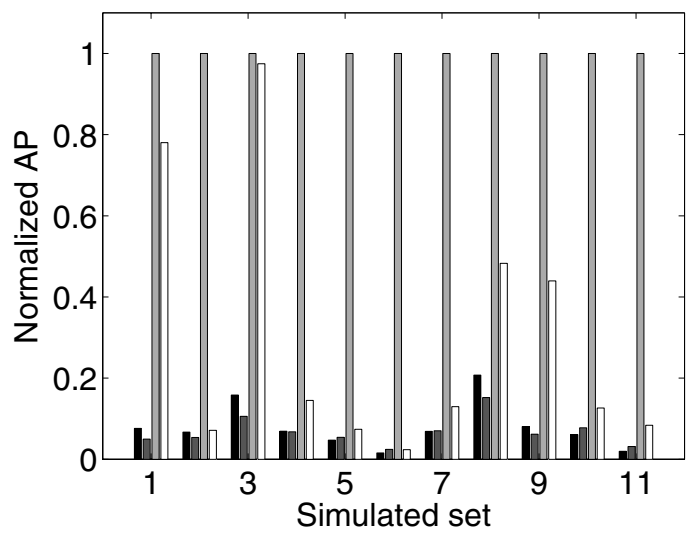

a) $\mathrm{AP}, \sigma_{l}^{2}=10^{-4}$

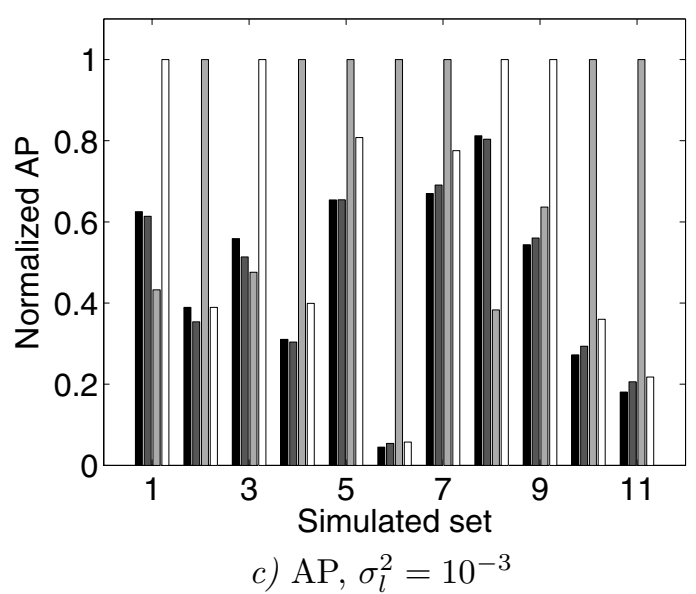

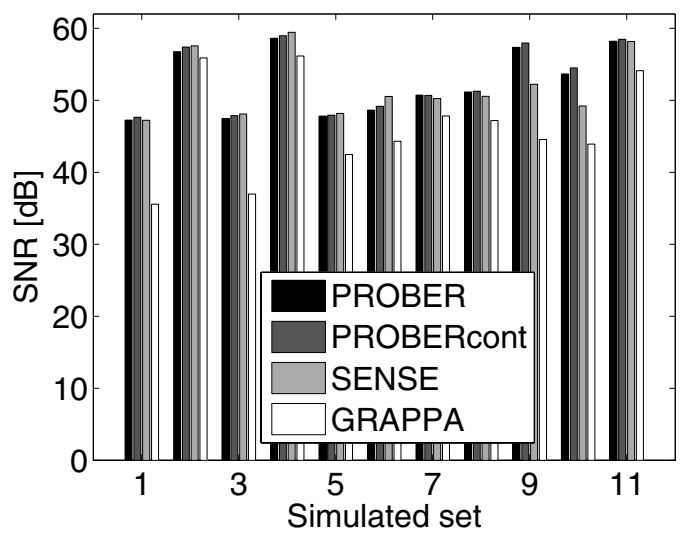

b) SNR, $\sigma_{l}^{2}=10^{-4}$

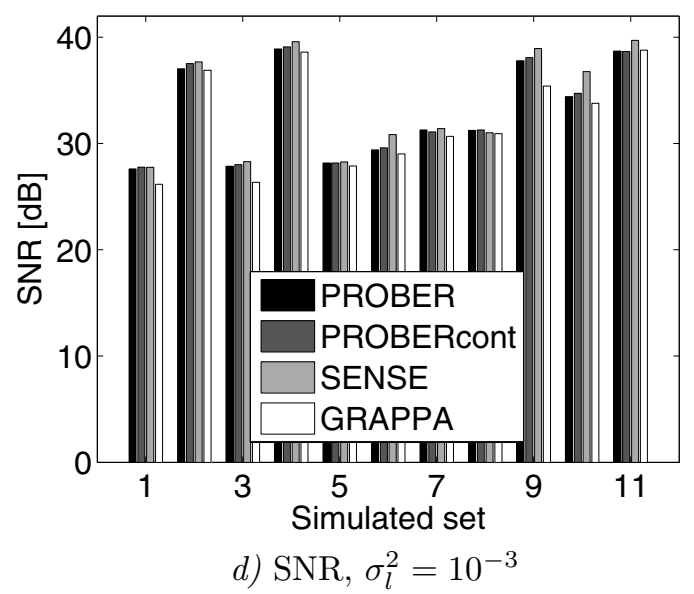

Figure 2. Reconstruction SNR and AP of discrete PROBER (black), continuous PROBER (dark gray), SENSE (gray) and GRAPPA (white) as compared on simulated images with noise variance (a), (b) $\sigma_{l}^{2}=10^{-4}$ and $(c),(d) \sigma_{l}^{2}=10^{-3}$. 


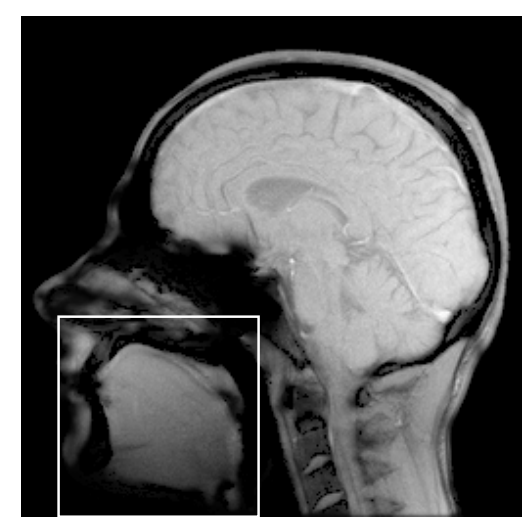

a) Original

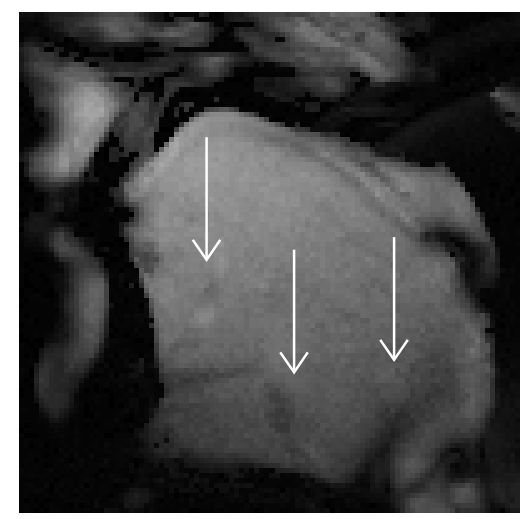

c) SENSE

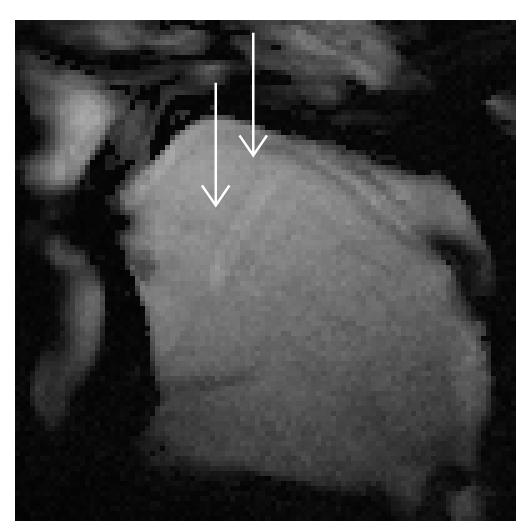

b) GRAPPA

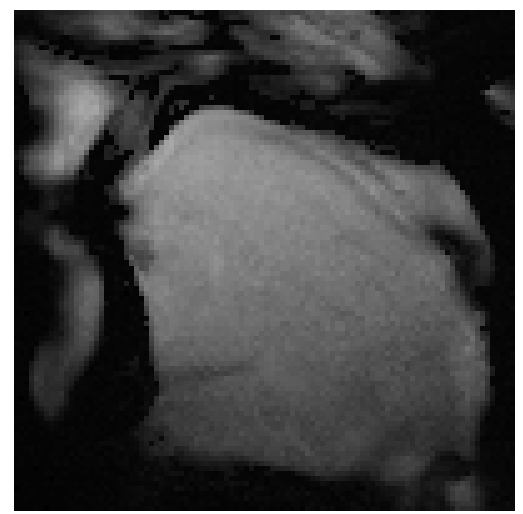

d) PROBER

Figure 3. The reconstruction of the simulated dataset 10 with acceleration factor 2. The original image is shown in (a). Magnified reconstructed images of (b) GRAPPA, (c) SENSE and (d) PROBER are shown. There are aliasing artifacts marked with arrows on the GRAPPA and SENSE reconstructions.

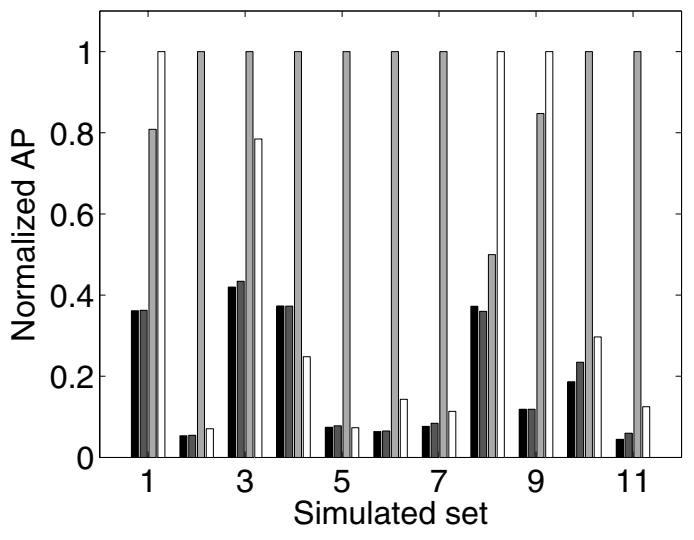

a) $\mathrm{AP}$

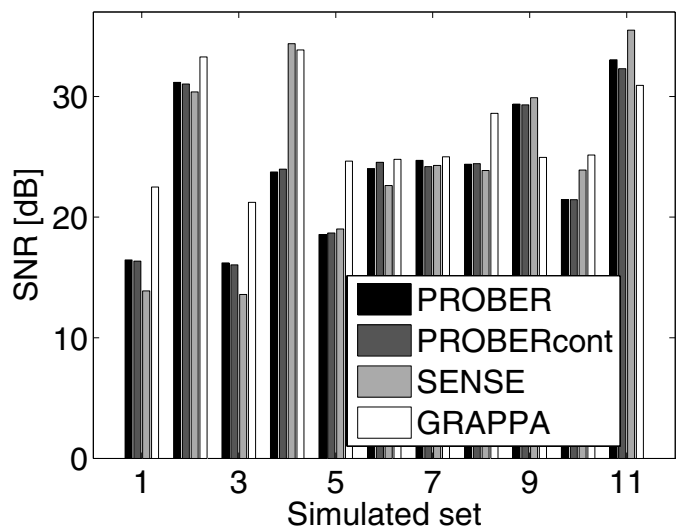

b) SNR

Figure 4. (a) Reconstruction AP and (b) SNR of discrete PROBER (black), continuous PROBER (dark gray), SENSE (gray) and GRAPPA (white) on simulated images with an acceleration factor 4 and noise variance $\sigma_{l}^{2}=5 \cdot 10^{-4}$. 


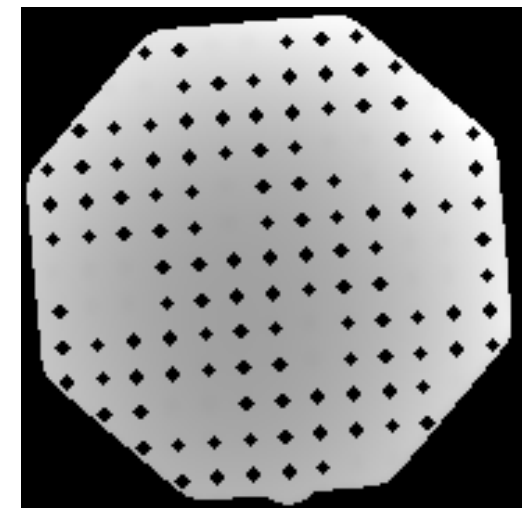

a) Original

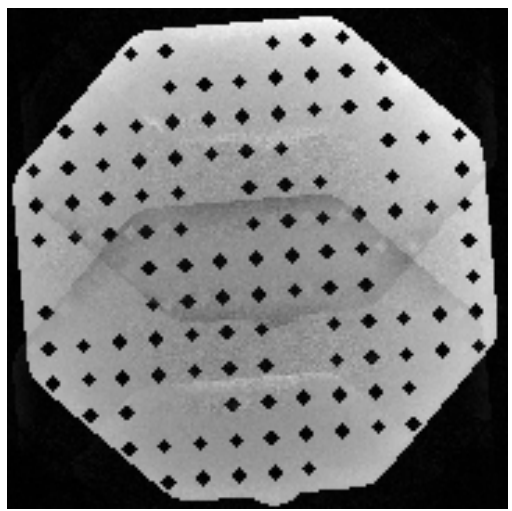

c) SENSE

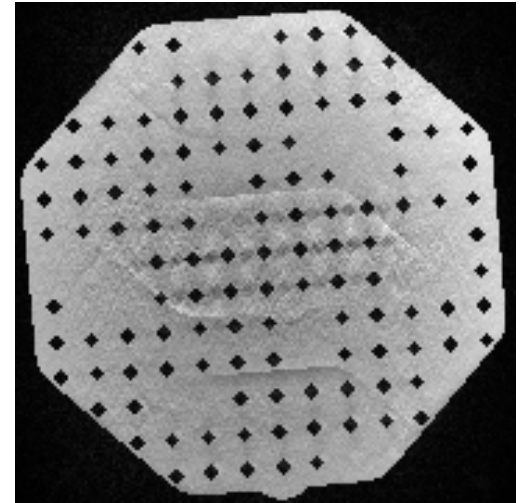

b) GRAPPA

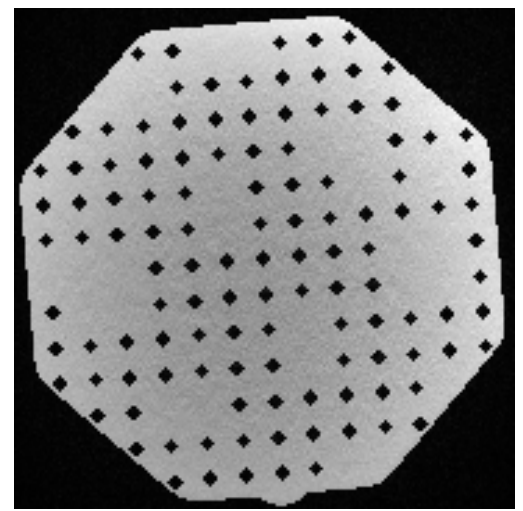

d) PROBER

Figure 5. The reconstruction of simulated set 9 with acceleration factor 4 . The original picture is shown in $(a)$. Images reconstructed using (b) GRAPPA, (c) SENSE and (d) PROBER are shown. The GRAPPA reconstruction is impaired with noise and significant aliasing artifacts. The SENSE reconstruction contains strong aliasing artifacts, whereas there is only moderate noise in the PROBER reconstruction with no aliasing artifacts.

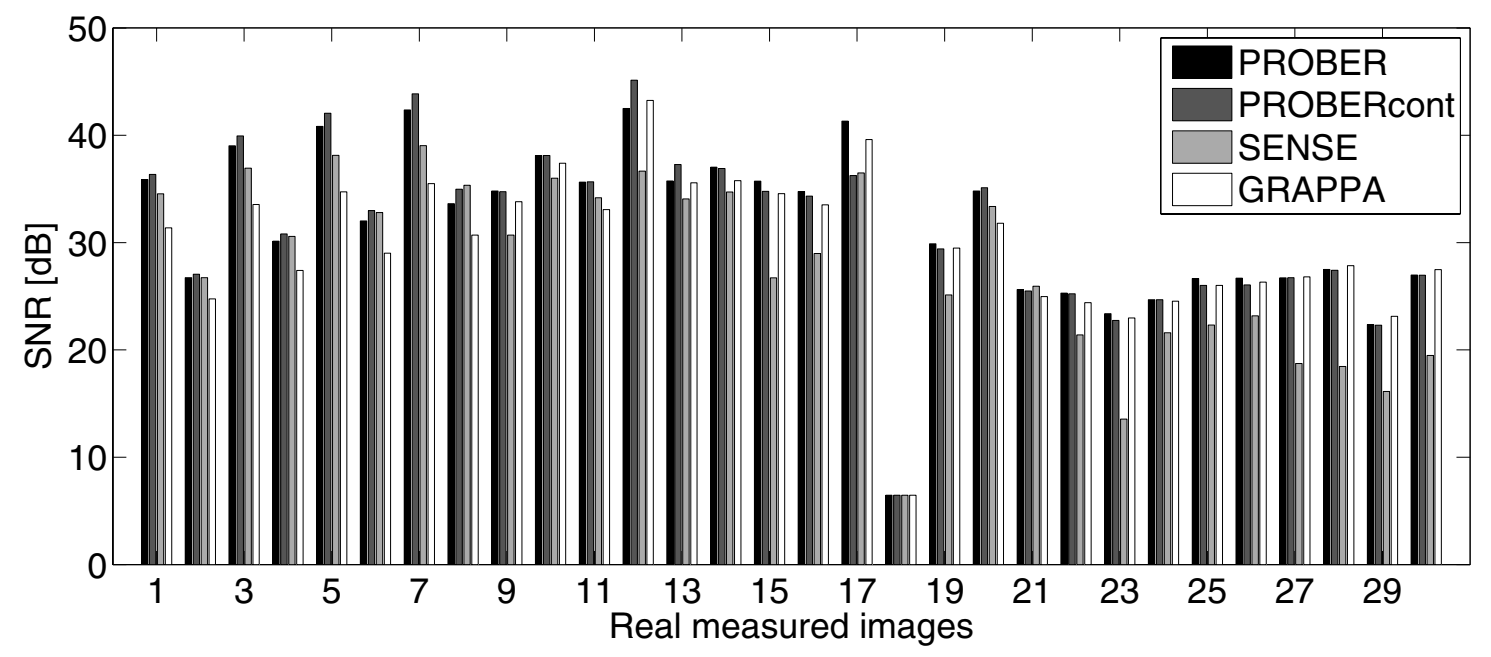

Figure 6. Reconstruction SNR as measured on the phantom (datasets 1-18) and in-vivo images (datasets 19-30). 


\section{CONCLUSION}

The PROBER method for parallel MRI reconstruction using B-spline approximation was enhanced by using a continuous norm to evaluate the reconstruction error. The method was tested and compared with two commerically available methods SENSE and GRAPPA. It was shown on simulated data that PROBER reaches better artifact suppression with the same level of noise in the reconstruction images especially for input images with small noise variance. The results were confirmed on real images. The PROBER reconstruction performs well also on images acquired with an acceleration factor 4 . The PROBER reconstruction offers a good alternative to commercially used methods as SENSE and GRAPPA.

\section{APPENDIX A. MINIMIZING THE RECONSTRUCTION ERROR}

The reconstruction error (9) is minimized by setting the partial derivatives $\frac{\partial e}{\partial g_{i j l}^{*}}$ to zero

$$
\begin{aligned}
\frac{\partial e}{\partial g_{i j l}^{*}=} & \sum_{m, m^{\prime}} \int_{x, y} \sum_{i^{\prime} j^{\prime} l^{\prime}}\left\{\sum_{p^{\prime} q^{\prime}} h_{p^{\prime} q^{\prime} l^{\prime}} \psi_{p^{\prime}, m^{\prime}} \psi_{q^{\prime}} g_{i^{\prime} j^{\prime} l^{\prime}} \varphi_{i^{\prime}, m} \varphi_{j^{\prime}} \sum_{p q} h_{p q l}^{*} \psi_{p, m^{\prime}} \psi_{q} \varphi_{i, m} \varphi_{j}\right. \\
& \left.-\delta_{m, m^{\prime}} \sum_{p^{\prime} q^{\prime}} h_{p^{\prime} q^{\prime}} \psi_{p^{\prime}, m} \psi_{q^{\prime}} \sum_{p q} h_{p q l}^{*} \psi_{p, m^{\prime}} \psi_{q} \varphi_{i, m} \varphi_{j}+\sigma_{l}^{2} g_{i^{\prime} j^{\prime} l^{\prime}} \varphi_{i} \varphi_{j} \varphi_{i^{\prime}} \varphi_{j^{\prime}}\right\} \mathrm{d} x \mathrm{~d} y \\
= & \sum_{i^{\prime} j^{\prime} l^{\prime}} g_{i^{\prime} j^{\prime} l^{\prime}}\left\{\sum_{p p^{\prime}} \int_{y} \sum_{m, m^{\prime}} \psi_{p^{\prime}, m^{\prime}} \varphi_{i^{\prime}, m} \psi_{p, m^{\prime}} \varphi_{i, m} \mathrm{~d} y \sum_{q q^{\prime}} h_{p^{\prime} q^{\prime} l^{\prime}} h_{p q l}^{*} \int_{x} \psi_{q^{\prime}} \psi_{q} \varphi_{j^{\prime}} \varphi_{j} \mathrm{~d} x+\sigma_{l}^{2} \varphi_{i} \varphi_{j} \varphi_{i^{\prime}} \varphi_{j^{\prime}}\right\} \\
& -\sum_{p p^{\prime}}\left(\sum_{m, m^{\prime}} \delta_{m, m^{\prime}} \int_{y} \psi_{p^{\prime}, m} \psi_{p, m^{\prime}} \varphi_{i, m} \mathrm{~d} y\right) \sum_{q q^{\prime}} h_{p^{\prime} q^{\prime}} h_{p q l}^{*}\left(\int_{x} \psi_{q^{\prime}} \psi_{q} \varphi_{j} \mathrm{~d} x\right) \\
= & \sum_{i^{\prime} j^{\prime} l^{\prime}} g_{i^{\prime} j^{\prime} l^{\prime} l^{\prime}}\left\{\sum_{p p^{\prime}} \Psi^{y}\left(p, p^{\prime}, i, i^{\prime}\right) \sum_{q q^{\prime}} h_{p^{\prime} q^{\prime} l^{\prime}} h_{p q l}^{*} \Psi^{x}\left(q, q^{\prime}, j, j^{\prime}\right)+\sigma_{l}^{2} \varphi_{i} \varphi_{j} \varphi_{i^{\prime}} \varphi_{j^{\prime}}\right\} \\
& -\sum_{p p^{\prime}} \Phi^{y}\left(p, p^{\prime}, i\right) \sum_{q q^{\prime}} h_{p^{\prime} q^{\prime}} h_{p q l}^{*} \Phi^{x}\left(q, q^{\prime}, j\right),
\end{aligned}
$$

where the B-spline kernels $\Psi^{x}, \Psi^{y}, \Phi^{x}$ and $\Phi^{y}$ are defined as

$$
\begin{aligned}
\Psi^{y}\left(p, p^{\prime}, i, i^{\prime}\right) & =\int_{y} \sum_{m, m^{\prime}=0}^{M-1} \psi_{p^{\prime}, m^{\prime}} \varphi_{i^{\prime}, m} \psi_{p, m^{\prime}} \varphi_{i, m} \mathrm{~d} y \\
\Psi^{x}\left(q, q^{\prime}, j, j^{\prime}\right) & =\int_{x} \psi_{q^{\prime}} \psi_{q} \varphi_{j^{\prime}} \varphi_{j} \mathrm{~d} x \\
\Phi^{y}\left(p, p^{\prime}, i\right) & =\sum_{m=0}^{M-1} \int_{y} \psi_{p^{\prime}, m} \psi_{p, m} \varphi_{i, m} \mathrm{~d} y \\
\Phi^{x}\left(q, q^{\prime}, j\right) & =\int_{x} \psi_{q^{\prime}} \psi_{q} \varphi_{j} \mathrm{~d} x .
\end{aligned}
$$

The B-spline kernels (16) are independent on the reference images and can be precomputed. The integration is done numerically with sufficiently small step.

The B-spline functions $\psi_{p}$ and $\psi_{q}$ are highly spatially localized (i.e. they are zero on most of its domain). Thus, the integral $\int_{x} \psi_{p}(x) \psi_{p^{\prime}}(x) \mathrm{d} x$ is zero for most of $p^{\prime} \neq p$. Therefore, the B-spline kernels (16) are sparse. Only the multiplications with non-zero kernel needs to be evaluated in (10). This reduces the complexity of the matrix evaluation in the continuous case nearly to $O\left(X^{2} I^{2} L^{2}+X I^{4} L^{2}\right)$ (assuming $I=J$ and $P=Q=X=Y$ in the low-resolution reference images). Thus, the time complexity of the continuous version of PROBER becomes equal to the complexity of the discrete version. 


\section{ACKNOWLEDGMENTS}

The authors were supported by The Grant Agency of the Czech Academy of Sciences under project 1ET101050403.

\section{REFERENCES}

1. D. K. Sodickson and W. Manning, "Simultaneous acquisition of spatial harmonics (SMASH): Fast imaging with radiofrequency coil arrays," Magnetic Resonance in Medicine 38, pp. 591-603, 1997.

2. M. A. Griswold, P. M. Jakob, R. M. Heidemann, M. Nittka, V. Jellus, J. Wang, B. Kiefer, and A. Haase, "Generalized autocalibrating partially parallel acquisitions (GRAPPA)," Magnetic Resonance in Medicine 47, pp. 1202-10, 2002.

3. K. P. Pruessmann, M. Weiger, M. B. Sheidegger, and P. Boesiger, "SENSE: Sensitivity Encoding for Fast MRI," Magnetic Resonance in Medicine 42, pp. 952-62, 1999.

4. M. A. Griswold, P. M. Jakob, M. Nittka, J. W. Goldfarb, and A. Haase, "VD-AUTO-SMASH imaging," Magnetic Resonance in Medicine 45(6), pp. 1066-74, 2001.

5. J. Petr, J. Kybic, S. Müller, M. Bock, and V. Hlaváč, "Parallel MRI reconstruction using B-spline approximation," in ISMRM '05: Proceedings of the 13th Scientific Meeting and Exhibition of International Society for Magnetic Resonance in Medicine, 13, p. 2421, International Society for Magnetic Resonance in Medicine, May 2005.

6. J. Petr, J. Kybic, V. Hlaváč, S. Müller, and M. Bock, "Fast parallel MRI reconstruction using B-spline approximation (PROBER)," in Proceedings of SPIE, M. J. Flynn and J. Hsieh, eds., Medical Imaging 2006: Physics of Medical Imaging, pp. 1251-1262, SPIE, February 2006.

7. M. Unser, "Splines: A perfect fit for medical imaging," in Proceedings of SPIE Symposium on Medical Imaging, 4684, SPIE, (San Diego, CA), February 2002.

8. D. K. Sodickson and C. A. McKenzie, "A generalized approach to parallel magnetic resonance imaging," Medical Physics 28, pp. 1629-43, August 2001.

9. M. Unser, "B-Spline Signal Processing: Part I - Theory," IEEE transactions on signal processing 41, pp. 821-32, February 1993.

10. M. Unser, "B-Spline Signal Processing: Part II - Efficient design and applications," IEEE transactions on signal processing 41, pp. 834-48, February 1993.

11. M. Unser, A. Aldroubi, and M. Eden, "Fast B-spline transforms for continuous image representation and interpolation," IEEE Transaction on Patter Analysis and Machine Intelligence 13, pp. 277-285, March 1991.

12. S. Horbelt, A. Muñoz, T. Blu, and M. Unser, "Spline kernels for continuous-space image processing," in Proceedings of the IEEE International Conference on Acoustics, Speech, and Signal Processing, June 2000.

13. W. Press, S. Teukolsky, W. Vetterling, and B. Flannery, Numerical recipes in C, Cambridge University Press, Cambridge, 2nd ed., 1992.

14. M. T. Vlaardingerbroek and J. A. Boer, Magnetic Resonance Imaging: Theory and Practice, Springer-Verlag, 1996.

15. J. G. Sled, A. P. Zijdenbos, and A. C. Evans, "A nonparametric method for automatic correction of intensity nonuniformity in MRI data," IEEE Transactions on Medical Imaging 17, pp. 87-97, February 1998.

16. C. A. McKenzie, E. N. Yeh, and D. K. Sodickson, "Improved spatial harmonic selection for SMASH image reconstructions," Magnetic Resonance in Medicine 46, pp. 831-6, 2001. 